\title{
Optoelectronic training program in industry: Philips Optoelectronics
}

\section{Bart Verbeek, Sander Arentsen}

Bart H. Verbeek, Sander Arentsen, "Optoelectronic training program in industry: Philips Optoelectronics," Proc. SPIE 3190, Fifth International Topical Meeting on Education and Training in Optics, (8 December 1997); doi: $10.1117 / 12.294391$

Event: Fifth International Topical Meeting on Education and Training in Optics, 1997, Delft, Netherlands 


\title{
Optoelectronic training programme in industry: Philips Optoelectronics
}

\author{
Bart H. Verbeek and Sander Arentsen \\ Philips Optoelectronics, Prof. Holstlaan 4, 5656 AA Eindhoven, The Netherlands \\ (email: arentsen@natlab.research.philips.com)
}

\begin{abstract}
The training programme that has been developed for operators, engineers and technicians in the factory and development department of Philips Optoelectronics will be described. Optoelectronics is a supplier of optoelectronic devices like semiconductor lasers, photodetectors and optical amplifiers for digital telecom, CATV and Multimedia Applications. The programme is based on small dedicated modules with a clear learning objective and all modules finish with a test. Different levels of training exist depending on the target group and required knowledge and skills. Most modules are made towards skills and daily practice. Optoelectronic device- and technology concepts are taught by experienced scientists from Philips Research and Development Departments.
\end{abstract}

\section{Introduction}

The market for optoelectronic products grows with $20 \%$ per year giving ample opportunities to optoelectronic component- and equipment suppliers to expand their business.

Philips Optoelectronics is a business unit of the Philips Group with semiconductor lasers, photodetectors and optical amplifiers for optical transmission and data storage and printing as products. Philips Optoelectronics is positioned to apply the knowledge and expertise of advanced compound semiconductor technology for offering high performance and innovative optoelectronic products for cable television, telecommunications and multimedia applications. To be successful in this rapidly growing market, well educated and trained people are essential both in the fabrication process ( operators and technicians ) as well as in the development ( product- and process engineers ) including application support engineers who have the ability to translate customers' needs into end solutions.

At Philips Optoelectronics, a training/education programme has been developed in order to meet the required level of skills and knowledge for the organisation. This means that different levels of training are required ranging from how to use a microscope, how to behave in a cleanroom and how to perform a specific production task to detailed knowledge of sophisticated processing steps and specification discussions with customers.

This paper describes briefly the outline of the training programme which is based on a CRI (Robert F. Mager and Peter Pipe: CRI-method (Criterion Referenced Instructions) ) philosophy. Small instruction steps are used which are always followed by a test. Individual programmes dedicated to specific tasks are therefore possible and the training speed and depth can be adjusted by the ambition of the individual worker. Performance testing is key. 


\section{Organisation of production}

In order to clarify some of the terms used in the programme, it is useful to introduce production lines (PL's). Four main groups can be distinguished in production lines at Philips Optoelectronics:

1: Waferfab: wafer- and chip fabrication including epitaxial growth of crystals and corresponding wafer/chip technology

2: Subassemblies: the production and preparation of parts needed to produce products like fibre pigtails, chip carriers and packages.

3: Assembly of coaxial products in $5.6 \mathrm{~mm}, 9 \mathrm{~mm}$ packages and photodiodes

\section{4: Assembly of Butterfly packaged lasers.}

Within the production lines, different Production Tasks (PT's) can be recognised that are very well described under ISO9001 requiring various machines, equipment and tools, e.g. bonding of gold wires to chips, cleaning steps, reflow soldering process and working with pick- and place machines. The production tasks are carried out by people with a specific job description. An operator who is able to perform many tasks within a production line (PL) gives high flexibility to the organisation.

Technicians for processes, testing, maintenance and shift leaders should be able to perform all production tasks at least within one production line. They also have a higher responsibility for SPC (Statistical Process Control), mentorship, trouble shooting and in quality process control.

Equipment engineers need to know all about the process- and characterisation equipment for which they are responsible. This requires knowledge of the production tasks, performance criteria of the equipment and also some knowledge of the final product specifications. Already here, a broader view is indispensable.

In the course design, one of the most important and time consuming activities is the analysis of a production task (PT). This is essential to define the optimum amount of knowledge required for the job with a minimum of training load. It is based on "what, how and with what" should the task be carried out. From the outcome, a clear educational programme with training modules can be defined.

At a higher level in the organisation of the production one find the department managers for logistics, engineering, waferfab and assembly/packaging. Training in business orientation and techniques, financial awareness and tools, managing techniques are relevant skills that are carried out by external training organisations.

\section{The Training Programme}

The CRI method is based on small training modules ( typically half day ) that need to be followed and completed. It is a self explanatory text with problems and solutions and stimulates the trainee to look for the right information and practice new skills. A mentor guides him/her and after completion of the module, a test is carried out. A personal training scheme exist for all employees to indicate the level of training/skills which is also used by local management to either stimulate further training or/and to optimise the production capacity. 


\section{Level Training Programme}

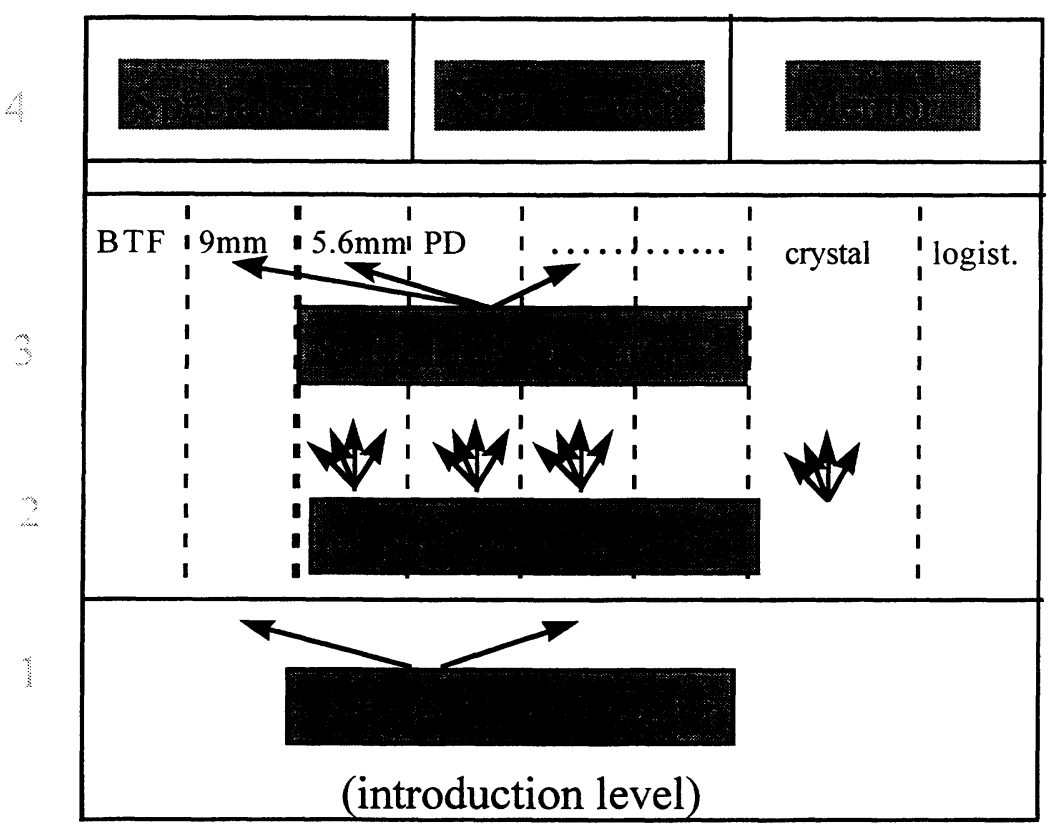

Figure 1: The Training programme model

Four levels exist in the plan for training ( see Figure 1) operators, which reflects the four levels of operation of employees. These are:

1: $\quad$ Single task operator

newly hired, "learning the ropes"

2: Multi task operator has completed level 1 but extended to more production tasks

(5) within the same production line.

3: Multi Line operator Multiple (5) production tasks but now in different $(2$ or more ) production lines.

4: $\quad$ Special Task Mentor requires very specialised (technical) skills for difficult jobs requires special social and communicative tasks and take Shift leader part in training programme social, communicative but also managerial skills.

A training programme exist for engineers with university degrees including introduction to Philips Optoelectronics, quality systems, ISO9001, ESD, product information, markets etc.. This will not be detailed further here. 


\subsection{Basic training level 1}

This training is made obligatory for operators as well as technicians within the first month. The programme has the following modules and lasts one week.

"Module" "content"

"Introduction"

parking place, toilet, lunchroom, working hours, boss

"product/market"

"employee"

"production task" tour of local facility, organisation products and market. local rules, labour contract, safety issues

"working environment" depends on task, see further under level 2 manuals cleanroom, clothing, ESD, working order, Quality system,

\subsection{Multi task Operator Level 2}

A multitask operator has successfully finished level 1 including a prime production task and also followed modules on other production tasks within the same production line. A production task usually requires both equipment task modules ( e.g. how to work with a microscope, bonding machine etc.) as well as the actual production steps and how to control the quality of this step.

An example will be given: If an operator is hired for chip bonding, he/she will follow the level 1 basic training first with a single production task "chip bonding". However before chip bonding can be done, it is necessary to pass the modules: "how to work with a microscope", "to work with proper chemicals", "cleaning the bondtool", "to work with bonding machine", before the operator is allowed to follow the training module "chip bonding". If on the other hand he/she will work on carrier assembly as a new task, the microscope, and chemical modules are still valid and only some additional modules are required. Thus, a personalised training path can be designed based on the individual ambitions as well as the department needs.

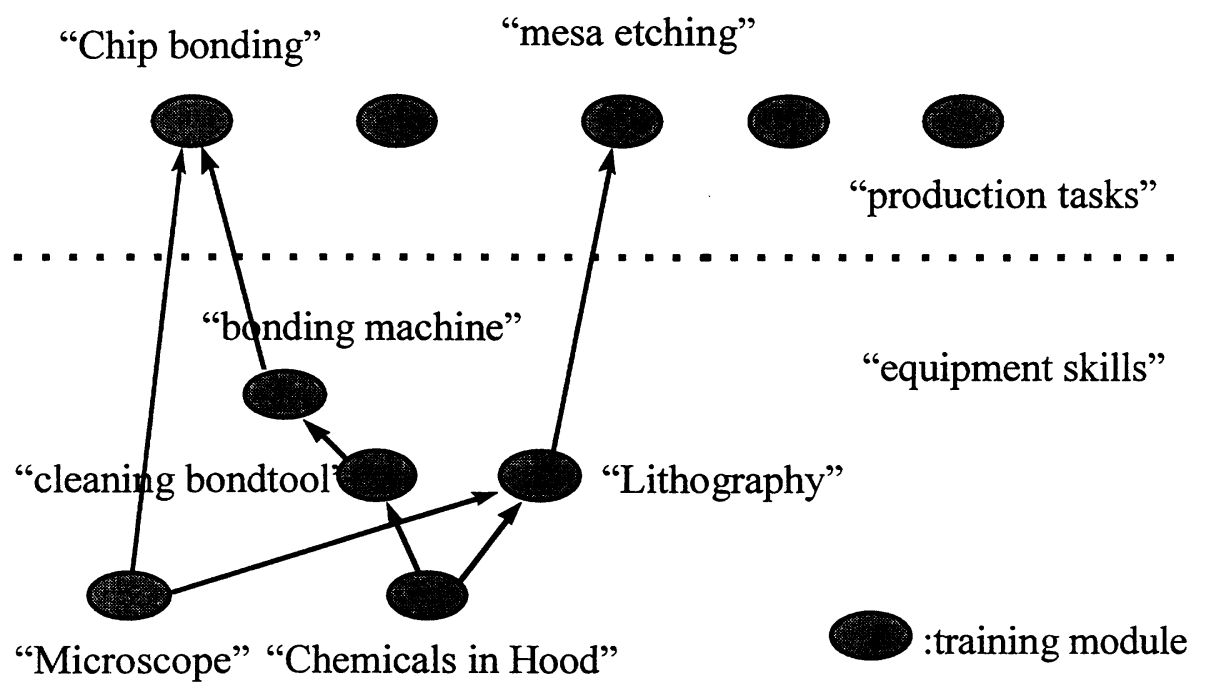

Levels 2 and 3 


\subsection{Multi line Operator Level 3}

$\mathrm{He} /$ She has passed many modules and is able to work at various sites and production lines to enhance flexibility of the organisation.

\subsection{Special task Level 4}

This level needs dedicated training modules with special mentor to perform a specialised technical job. For mentor and shiftleader tasks, external training programmes are used.

\section{Training Modules}

All modules have the same structure and layout and start with a description of the goal of the module and the final test criteria for clarity. Then the "why" of the module is described in some detail, "how" does the equipment/process look/work like with relevant details, definition of terms and an actual description of how to perform the task. At the end a number of exercises are given that can be carried out in the presence of the mentor. The module is considered as a tool for the operator to achieve the goals described at the beginning of the module. This implies that no reading or exercise is obligatory. The test on the other hand, with which the operator proves that performance is at the desired level, is obligatory. This gives operators the opportunity to spend their training time as efficient as possible. With the responsibility for achieving the goals given to themselves, operators can decide which parts are requiring full attention and exercising and which parts not.

If the test is successful, the result will be marked on the personal training card. A certificate will be handed out when all modules for the individual levels have been passed.

\section{Training Programme for Engineers}

Since no specific training programme is available for engineers and developers in the university educational programmes, a dedicated course has been developed by senior research- and developing staff members of Philips Optoelectronics. This course has 12 modules each taking 2 hours of presentation including daily working examples and are focused on the products and processes that are carried by Optoelectronics. Therefor the content of the course is very specific and cannot be described in great detail. The target level is a university degree in which the basic foundations like waveguiding, semiconductor physics, measuring equipment are taken for known. In addition, further information on detailed product and production processes are given.

The following modules are used:

Physics of semiconductor lasers:

Characterisation

Crystal Growth

Technology, Metallisation

\section{Coating}

Packaging

Reliability basic rate eqs., DFB lasers, modulation properties etc. Strained MWQ's, Measuring techniques, farfield, nearfield, etc epitaxy, crystalstructures, defects, LPE, MOVPE, VPE, MBE.

Lithography, Wet- and Dry etching, Deposition techniques, implantation, chip separation and mounting, electrical properties and chemistry of metallisation schemes.

Effect of coatings on reflectivity, protection coatings, different coat processes Optical properties, thermal effects, RF, mechanical, lenses, isolators, also technology Statistics, measuring lifetime and its conditions, burn-in, stress and lifetime tests. Also product reliability. 
Degradation Mechanisms

Failure Analysis, what can be learned from SEM.

Fibre-optic Products and Applications Specialised information

Visible lasers and Applications

Specialised information

Photodiodes

Physics, responsivity, speed, frequency response

The emphasis on certain topics may become stronger for those areas of topics that the student need to follow for executing his daily job.

\section{Conclusion}

This paper has described the training programme of Philips Optoelectronics both for operators as well as for engineers that need specific job information to perform a well defined production task or to give basic background level information for engineers. The concept of modules in the various levels of operation has proven to be very successful and the method will be further developed and improved on the level of the modules. This training programme has been used for newly hired people in the factory for operator and technician that are able to participate in the full production process in a very efficient and fast way. 